\title{
Uncertainties in the Position of the $\beta$ Cephei Instability Strip in the HR Diagram
}

\author{
A.A. Pamyatnykh, W.A. Dziembowski, P. Moskalik \\ Copernicus Astronomical Center, Polish Academy of Sciences \\ ul. Bartycka 18, 00-716 Warsaw, Poland
}

\begin{abstract}
We discuss the sensitivity of the theoretical B star instability domains to the heavy element abundance $Z$, the adopted metal mixture, the assumed overshooting from stellar convective cores and the choice of the opacity data.
\end{abstract}

A very good agreement between results of stability surveys (Dziembowski \& Pamyatnykh, 1993; Gautschy \& Saio, 1993; Dziembowski, Moskalik, \& Pamyatnykh, 1993) conducted for models employing the OPAL opacities (Iglesias, Rogers, \& Wilson, 1992) with observational data on B type pulsators leaves no doubt that the long searched driving mechanism has been correctly identified. This is the usual $\kappa$ mechanism acting in the layer at $T \approx 2 \times 10^{5} \mathrm{~K}$ where a pronounced bump in the metal opacities occurs. The good agreement concerns not only $\beta$ Cep stars but also SPB (53 Per) stars which are B3-B9 type objects exhibiting light variations with periods of 1 to $4 \mathrm{~d}$. The whole domain in the H-R diagram where the instability driven by the metal opacity bump occurs we call the $\beta$ Cephei instability strip. This is in an analogy with the $\delta$ Cep instability strip, where stellar pulsations are driven by the opacity mechanism acting in the Hell ionization zone.

The purpose of our research was to examine prospects for using occurrence of $B$ type pulsators in a stellar system as a diagnostic of the system properties as well as a test of the stellar evolution theory. The main advantage over the similar efforts in the case of $\delta$ Cep instability strip is that the convection plays no role in $\mathrm{B}$ star pulsations. We conduct a similar stability survey to those described in our earlier papers. However, in addition to original OPAL opacities, we use their updated version as well as OP (Seaton et al. 1993) data, which allowed us to assess uncertainties introduced by various approximations adopted in the opacity calculations. Moreover, we considered cases of lower metal abundance and models with convective overshooting.

The position of the Blue Edge of the $\beta$ Cephei instability domain is determined by the metallicity, primarily by the abundance of the iron-group elements ( $\mathrm{Fe}$, but also $\mathrm{Cr}, \mathrm{Mn}, \mathrm{Ni}$ ). An observational determination of the Blue Edge in a stellar system, therefore, yields a probe of the system's metallicity. With the OP tables $Z=0.015$ is sufficient to explain the excitation of almost all cluster $\beta$ Cephei variables. With the original OPAL data the corresponding $Z$ values was above 0.02 . Most recent data, based on calculations with additional 7 elements included, lower that number but still there is some 0.003 difference in the inferred $Z$-value depending on the choice of the opacity tables (OP vs. OPAL). 
The instability domain extends to red well beyond the Main Sequence band. However, since the Post-Main Sequence evolution is very fast we identify the red edge of the instability strip with that of the MS band (TAMS). Position of TAMS in the HR diagram depends on the overall metallicity $Z$ and on the amount of the convective overshooting assumed in the models. Both, increase in $Z$ and the overshooting distance moves TAMS toward red. The latter effect is more significant. The observed position of the red edge constrains the overshooting length. Standard models without convective overshooting explain very well the position of the $\beta$ Cephei stars in the HR diagram. On the contrary, allowing even small overshooting results in a sizable extension of the instability domain toward lower $T_{\text {eff }}$ and longer periods, where no $\beta$ Cephei stars are found.

With decreasing $Z$ the $\beta$ Cephei instability domain shrinks down and finally vanishes. In the case of OP opacities this happens at $Z \approx 0.012$.

The observed range of $\beta$ Cep star luminosities and corresponding masses is significantly narrower than that predicted by the theory. In our survey we found that the short-period mode instability begins at $M \approx 7.5 M_{\odot}$, while the minimum mass for the observed $\beta$ Cep stars is about $9 M_{\odot}$. We do not know whether this discrepancy reflects some inadequacy in the stellar opacity calculation or a difference in the nonlinear development of the instability.

There is no upper luminosity limit of the instability domain. Kiriakidis, Fricke \& Glatzel (1993) showed that the blue edge, which initially moves away from ZAMS with mass increase, at $M \approx 20 M_{\odot}$ turns back and reaches it at about $70 M_{\odot}$. We have confirmed this result and found that the instability strip is the narrowest in terms of $T_{\text {eff }}$ at $M \approx 18 M_{\odot}$. In Sterken \& Jerzykiewicz (1993) list of $\beta$ Cep stars there is only one (V986 Oph) occuring in the upper part of the instability domain. The apparent paucity of hot and luminous $\beta$ Cep calls for explanation.

The high order $\mathrm{g}$-mode instability occurs in a wide range of temperatures and it is quite robust. It persists at lower metal abundance $(Z \approx 0.006$ for the OP opacities) than that of the p modes excited in $\beta$ Cep stars. The use of the OP instead of OPAL opacities promotes the instability, extending it to somewhat higher degrees (longer periods).

Acknowledgments. This research was supported by KBN grant Nr 2 P304 013 07. WAD and PM gratefully acknowledge travel grants from the IAU.

\section{References}

Dziembowski, W. A., Moskalik, P., \& Pamyatnykh, A. A. 1993, MNRAS, 265, 588

Dziembowski, W. A., \& Pamyatnykh, A. A. 1993, MNRAS, 262, 204

Gautschy, A., \& Saio, H. 1993, MNRAS, 262, 213

Iglesias, C. A., Rogers, F. J., \& Wilson, B. G. 1992, ApJ, 397, 71

Kiriakidis, M., Fricke, K. J., \& Glatzel, W. 1993, MNRAS, 264, 50

Seaton, M. J., Yu Yan, Mihalas, D., \& Pradhan, A. K. 1994, MNRAS, 266, 805

Sterken, C,. \& Jerzykiewicz, M. 1993, Space Sci. Rev., 62, 95 